\title{
NASA's In-Space Propulsion Technology Program: Overview and Update
}

\author{
Les Johnson, ${ }^{*}$ Leslie Alexander, ${ }^{\dagger}$ Randy M. Baggett,,$\neq$ Joseph A. Bonometti, ${ }^{\S}$ Melody Herrmann, \\ Bonnie F. James," and Sandy E. Montgomery ${ }^{* *}$ \\ NASA Marshall Space Flight Center \\ Huntsville, Alabama 35812
}

\begin{abstract}
NASA's In-Space Propulsion Technology Program is investing in technologies that have the potential to revolutionize the robotic exploration of deep space. For robotic exploration and science missions, increased efficiencies of future propulsion systems are critical to reduce overall life-cycle costs and, in some cases, enable missions previously considered impossible. Continued reliance on conventional chemical propulsion alone will not enable the robust exploration of deep space-the maximum theoretical efficiencies have almost been reached and they are insufficient to meet needs for many ambitous science missions currently being considered. The In-Space Propulsion Technology Program's technology portfolio includes many advanced propulsion systems. From the next-generation ion propulsion system operating in the 5- to $10-\mathrm{kW}$ range to aerocapture and solar sails, substantial advances in spacecraft propulsion performance are anticipated. Some of the most promising technologies for achieving these goals use the environment of space itself for energy and propulsion and are generically called "propellantless" because they do not require onboard fuel to achieve thrust. Propellantless propulsion technologies include scientific innovations such as solar sails, electrodynamic and momentum transfer tethers, aeroassist, and aerocapture. This paper will provide an overview of both propellantless and propellant-based advanced propulsion technologies, as well as NASA's plans for advancing them as part of the In-Space Propulsion Technology Program.
\end{abstract}

\section{Introduction}

The In-Space Propulsion Technology Program is entering its third year and significant strides have occurred in the advancement of key transportation technologies that will enable or enhance future robotic science and exploration missions. At the program's inception, a set of technology investment priorities were established using an Agencywide prioritization process and, for the most part, has changed little, thus allowing a consistent framework in which to fund and manage technology development. The technology priorities are listed in Table 1. High-priority technologies are those considered enabling for a set of high-value midterm science targets. Medium-priority technologies are those that will enhance or enable missions that are not perceived as critical as those benefiting from the high-priority technologies, but are still important and need significant funding. Low-priority technologies will be matured as funding is available. The high-payoff/high-risk technologies are those that are the most immature, yet have the potential to be considered high priority once some key science and engineering feasibility questions are answered.

\footnotetext{
* Manager, In-Space Propulsion Technology Projects Office, TD05

$\dagger$ Technology Area Manager, Chemical and Thermal Propulsion Project, TD05

‡ Technology Area Manager, Next-Generation Electric Propulsion Project, TD05

\& Technology Area Manager, Emerging Propulsion Technologies, TD05

I Manager, Technology Planning and System Analysis, TD05

\# Technology Area Manager, Aerocapture Technology Project, TD05

*** Manager, Solar Sail Propulsion Project, TD05
} 
Technology development within the In-Space Propulsion Technology Program is funded primarily through competitive NASA Research Announcements and with industry, universities, and NASA field Centers eligible to bid. The result has been a national effort focused on maturing in-space propulsion technologies to technology readiness level (TRL) 6 for transition to flight at the earliest possible date.

Table 1. In-Space Propulsion Technology Program Priorities

\begin{tabular}{|l|l|l|l|}
\hline \multicolumn{1}{|c|}{ High Priority } & \multicolumn{1}{|c|}{ Medium Priority } & \multicolumn{1}{c|}{ Low Priority } & \multicolumn{1}{c|}{ High Payoff/High Risk } \\
\hline Aerocapture & Advanced chemical & Solar thermal & $\begin{array}{l}\text { Momentum exchange } \\
\text { electrodynamic reboost tethers }\end{array}$ \\
\hline $\begin{array}{l}\text { Next-generation } \\
\text { solar electric } \\
\text { propulsion }\end{array}$ & $\begin{array}{l}\text { Solar electric propulsion } \\
\text { (including Hall thrusters) }\end{array}$ & & $\begin{array}{l}\text { Very low areal density } \\
\text { solar sails }\end{array}$ \\
\hline Solar sails & & & \\
\hline
\end{tabular}

\section{Aerocapture}

\section{A. General Description}

Aerocapture uses a planet's atmosphere to accomplish a quick, near-propellantless orbit capture-the placement of a space vehicle in its proper orbit. The atmosphere is used as a brake to slow down a spacecraft, transferring the energy associated with the vehicle's high speed into thermal energy.

The aerocapture maneuver starts with a hyperbolic trajectory into the atmosphere of the target body. The atmosphere's density creates friction, slowing the craft and placing it into an elliptical orbit. Onboard thrusters are then used to circularize the orbit.

This nearly fuel-free method of decelerating a space vehicle could reduce the typical mass of an interplanetary spacecraft by more than half, allowing for a smaller and less expensive vehicle-one better equipped to conduct long-term science at its destination and to enable greater scientific return - and for faster trip times. In fact, aerocapture, when combined with solar electric propulsion technology, enables missions to outer planet destinations that would be impractical using conventional propulsion.

The requirement to slow down a spacecraft nonpropulsively can be achieved in two ways. The craft can be enveloped by a structure with heat shielding applied to the external surfaces. Such rigid "aeroshells" were used during entry and descent into Mars' atmosphere by the Mars Pathfinder in 1997 and the Mars Exploration Rovers in early 2004. However, one distinction is that these missions were direct entry into a planetary atmosphere while aerocaptured systems will remain in orbit. Another option is for the vehicle to deploy an aerocapture inflatable deceleration system as an inflatable ballute - a combination parachute and balloon made of thin, durable material.

\section{B. Technical Approach}

Four different technology concepts are under consideration or development for aerocapture use: (1) blunt body, rigid aeroshell design; (2) slender body, rigid aeroshell design; (3) trailing ballute design; and (4) attached ballute design.

The blunt body, rigid aeroshell system encases a spacecraft in a protective shell. This shell provides an aerodynamic control surface and a means of protection from the high heating experienced during high-speed atmospheric flight. Once a space vehicle is captured into a planet's orbit, the aeroshell is jettisoned.

An aeroshell consists of three main parts: (1) the external thermal protection material; (2) adhesives, which are used to bond the thermal protection system (TPS) to the aeroshell; and (3) an underlying structure to which the internal spacecraft and the external thermal protection material are attached. The challenges with this approach are to customize the design and thickness of the thermal protection material to accommodate different heating characteristics endured during aerocapture, and to develop adhesives capable of withstanding extremely high temperatures. 
The slender body configuration looks much like an elongated capsule, with a hard shell surrounding the spacecraft. The design could provide increased volume in the interior of the spacecraft when compared to the blunt body design, allowing for improved packaging of larger crafts. Because of its slender body shape, the system also could provide increased tolerance for navigational and atmospheric uncertainties.

The trailing ballute features an inflated toroidal volume that is much larger than the spacecraft it is towed behind, much like a parachute, to slow the vehicle down. The trailing ballute design allows for easy detachment and minimizes interference with the spacecraft's operation. The ballute itself is made of a lightweight, thin-film material.

The trailing ballute design may have performance advantages over the aeroshell design. One such advantage is not enclosing the payload in a rigid shell during interplanetary cruise. Not having an enclosure around the spacecraft allows it to take full advantage of the volume available in the launch vehicle shroud. Also, in an aeroshell design, the shell takes all the force and heating of reentry. But with the trailing ballute design, the ballute would incur most of the aerodynamic forces and heat, allowing any protection around the payload to be very lightweight.

The attached forebody ballute looks much like the aeroshell or blunt body. The attached ballute is often referred to as a hybrid system, with a rigid foreshell and an inflated, attached ballute extending from either the front or back of the spacecraft.

The inflatable, attached ballute extends from a rigid nosecap and works much like a parachute, providing a large surface area to slow the spacecraft down to allow for an aerocapture maneuver to occur. As the spacecraft approaches a planet's atmosphere, the ballute is inflated and then jettisoned once the craft is captured into orbit.

Made of thin, lightweight material, such as DuPont Kapton ${ }^{\circledR}$, the inflatable, attached ballute design offers many of the same advantages and functionality as trailing ballute designs. The challenge facing researchers is to create a flexible, inflatable TPS to protect the spacecraft from the intense heat experienced during atmospheric entry.

\section{Status}

Aerocapture has never been flight tested. Relevant experience, however, exists from ablative entry capsules. Ablative entry technologies have been used throughout the history of the U.S. Space Program, including the Apollo return capsule and the Galileo probe.

\section{Recent Accomplishments}

In 2003 and 2004, several aerocapture TPS candidate materials, both ablative and nonablative, have undergone extensive arcjet testing. Advanced, lightweight structures have also been developed and are currently undergoing testing. Applied Research Associates, NASA Ames Research Center, NASA Langley Research Center (LaRC), and Lockheed Martin are involved in TPS/structures development for rigid aeroshell aerocapture applications. Both heat flux and recession sensors are also being developed at Ames Research Center. These sensors have been integrated into TPS and arcjet tested.

In 2003, Ball Aerospace made significant progress in the design, development, and testing of a trailing ballute system. Progress was made in the areas of ballute shape, survivability, flow stability, aeroelastic response and separation. Lockheed Martin won a contract to develop an inflatable aeroshell concept and Ball Aerospace won a contract for an inflatable attached afterbody concept. In 2002, the In-Space Propulsion Technology Program completed a systems definition study of aerocapture at Saturn's moon, Titan. Results showed significant reductions in trip time and overall system mass by utilizing aerocapture versus all-propulsive capture. The aerothermal analyses also uncovered that radiative heating accounted for a significant portion of the overall heating to the aerocapture vehicle. Technology development tasks were then initiated to accommodate those findings. In 2003, a systems definition study of aerocapture at Neptune was completed. Results of that study showed that aerocapture was a feasible approach and would save trip time and overall mass. In 2004, the In-Space Propulsion Technology Program is conducting a systems study of aerocapture at Venus.

In the summer of 2003, aerocapture was selected as one of five candidate technologies for the New Millennium Program ST-9 mission. ST-9 would provide an opportunity to flight validate aerocapture computational modeling and design tools and would provide for an opportunity to flight validate an integrated aerocapture system for future mission applications. 


\section{Next-Generation Solar Electric Propulsion}

\section{A. General Description}

Solar electric propulsion (SEP) uses solar array power to ionize and accelerate heavy propellants, such as xenon, as inputs to a low-thrust, fuel-efficient, ion propulsion system (IPS). The typical IPS consists of ion thrusters, power processor units, and a propellant storage and management subsystem. The low thrust level of ion engines means that they have to run for a long time to accelerate the spacecraft to its desired velocity. For example, if the ion engine on Deep Space 1 (DS 1), NASA Solar Electric Propulsion Technology Application Readiness (NSTAR), was continuously operated at its maximum thrust, the spacecraft would accelerate from zero to $60 \mathrm{mph}$ in $\approx 40 \mathrm{hr}$. However, after a year of thrusting at this level the spacecraft would be traveling more than $10,000 \mathrm{mph}(>4,500 \mathrm{~m} / \mathrm{s})$. In addition, ion engines are the most fuel-efficient rockets used in space today. They are roughly 10 times more fuel-efficient than conventional chemical rocket engines.

\section{B. Technical Approach}

The simplest form of SEP, electrothermal thrusters, uses the heat produced from electric current passing through resistance heaters or the propellant itself to energize the propellant. Electrothermal thrusters have seen broad application on commercial satellites in recent years for stationkeeping and attitude control. It is a mature technology and has a rich future for applications involving orbit transfer and relatively high thrust.

The second type of SEP, electrostatic thrusters, relies on application of an electric field to accelerate the propellant directly. The propellant is initially ionized and then injected across a voltage potential established between an anode and cathode. The resulting force on the charged propellant ions accelerates them to high exhaust velocities. This type of device can achieve very high specific impulse (3,000 to $12,000 \mathrm{~s}$ and above) and is ideal for missions in which propellant mass must be minimized. These devices are generally limited to very low thrusts, but can achieve extremely high vehicle velocities due to their efficient propellant utilization. Ion propulsion and Hall thrusters fall into this category.

The third type of SEP, electromagnetic thrusters, employs a combination of electric and magnetic fields to accelerate ionized propellant and produce thrust. There is a wide variety of options available involving steady and pulsed modes of operation. All rely on the Lorentz force arising from the flow of ions (current) through a magnetic field to accelerate the propellant. These devices are more limited in terms of specific impulse than ion propulsion, but they are able to process higher amounts of power. These types of systems are ideal for applications where higher thrust levels are required. Magnetoplasmadynamic thrusters, Lorentz force accelerators, and pulsed inductive thrusters fall into this category.

The emphasis of the In-Space Propulsion Technology Program is on ion thrusters, though Hall thrusters are also part of the technology portfolio.

\section{Status}

Ion engines have been demonstrated in space and are the primary propulsion system for NASA's Dawn mission. The first use of an ion propulsion system for primary propulsion was on the DS 1 mission in 1998.

The ion engine on DS 1, jointly developed by NASA Glenn Research Center (GRC), Boeing, and the NASA Jet Propulsion Laboratory (JPL), was designed to operate for $1 \mathrm{yr}$ at its maximum power level of $2.5 \mathrm{~kW}(\approx 3.4 \mathrm{hp})$. Over this time NSTAR used $\approx 83 \mathrm{~kg}$ of xenon, the propellant of choice for ion thrusters. Xenon is found naturally in Earth's atmosphere, is chemically inert, and environmentally safe. Several long duration tests were performed to make sure the ion engine for DS 1 would last long enough to perform the mission. These included a 1,000-hr test, a 2,000-hr test, and an 8,000-hr test, all performed at the engine's maximum power level. The maximum power level is believed to be the most stressing condition for the engine. Beginning in the fall of 1998, the DS 1 flight spare engine was placed in a long duration test at JPL with the objective of demonstrating that the engine could be run for $150 \%$ of its design life. At the end of the JPL extended life test in June 2003, the NSTAR flight spare engine had operated for more than $30,352 \mathrm{hr}$ and processed more than $235.1 \mathrm{~kg}$ of xenon. This is by far the longest any rocket engine has ever been operated and corresponds to $283 \%$ of its original design life.

\section{Recent Accomplishments}

The next-generation electric propulsion (NGEP) focus is to advance electric propulsion system and component technologies. In the past year under NGEP, GRC completed phase I of NASA's evolutionary xenon thruster (NEXT) development. NEXT phase I objectives included definition of the NEXT propulsion system requirements 
and a successful demonstration of system level performance at power levels in excess of $7 \mathrm{~kW}$ using an engineering model NEXT thruster with a breadboard power processing unit and breadboard propellant management system.

GRC also operated the NEXT $40-\mathrm{cm}$ thruster for more than $2,000 \mathrm{hr}$ and identified several design improvements for the prototype thruster currently under development in phase 2 . JPL's extended life test (ELT) of the DS 1 flight spare NSTAR thruster exceeded $30,000 \mathrm{hr}$ of operation before completing a planned shutdown. At its conclusion in June 2003, the NSTAR ELT was the longest life test ever performed on an ion thruster. Following the ELT, thruster life test data and hardware evaluation significantly contributed to improving the NEXT thruster design. Under the NGEP carbon-based ion optics effort, JPL successfully completed design, fabrication, vibration and performance testing of 30-cm carbon-carbon ion optics. Boeing Electron Dynamic Devices completed fabrication and vibration test of 30-cm pyrolytic graphite grids. If flight qualified, these grids will significantly improve the life/throughput capability of ion thrusters. Other NGEP significant technology advancements were made by GRC in high power Hall thruster and high current hollow cathode designs. GRC completed performance testing on the NASA-457M Hall thruster up to $90 \mathrm{~kW}$ supporting further development of an engineering model thruster.

\section{Solar Sails}

\section{A. General Description}

Solar sail propulsion uses sunlight to propel vehicles through space by reflecting solar photons from a large, mirror-like sail made of a lightweight, reflective material. This source of propulsion is not to be confused with the solar wind, which does not provide any significant level of thrust. The continuous photonic pressure provides propellantless thrust to hover indefinitely at points in space or conduct orbital maneuver planes changes much more efficiently than conventional chemical propulsion. Eventually, it might propel a space vehicle to tremendous speeds - theoretically much faster than any present-day propulsion system. Because the Sun supplies the necessary propulsive energy, solar sails also require no onboard propellant, thus reducing payload mass.

First-generation sails will vary in size from 100 to $200 \mathrm{~m}$, depending on mission destination, and typically would be three-axis stabilized. It would be compacted and stowed for launch. Once deployed, the sails would be supported by ultra-lightweight trusses.

Solar sails are composed of flat, smooth material covered with a reflective coating and supported by lightweight structures attached to a central hub. Near-term sails likely will use aluminized DuPont Mylar ${ }^{\circledR}$ or CP1. Both materials have previously flown in space. More robust sails might use a meshwork of interlocking carbon fibers.

\section{B. Technical Approach}

A solar sail could fly in low-Earth orbit, but it would need to be robust enough to withstand significant gravity and environmental loads. Missions to the outer solar system and beyond require further innovations in architectures and materials. While an interstellar probe is a notable potential future mission application for sails, it is a far-term vision. The most near-term applications are for heliocentric missions in the Earth's neighborhood (the Earth-Sun L1 libration point for example). This class is the current focus of the In-Space Propulsion Technology Program's effort. It will lead to the next generation of solar sails which will enable a close approach to the Sun $(<0.25 \mathrm{au})$ where thermal and radiation environments will be more stressing.

NASA is concentrating its development effort on the three-axis stabilized, square sails. This sail looks much like a kite. Four booms extend from a central hub that houses the four triangular sail quadrants during launch.

\section{Status}

Two teams have been selected by NASA to lead hardware development activities that will culminate in ground demonstrations of key solar sail technology systems. L'Garde, Inc., of Tustin, CA, is developing a solar sail system that employs booms that are flexible at ambient temperatures but "rigidize" at temperatures below $-35^{\circ} \mathrm{C}$. Their concept uses articulated vanes located at the corners of the square to control the solar sail attitude and thrust direction. Able Engineering Company of Goleta, CA, is developing a coilable longeron that deploys in space much the way a spring-loaded screw is rotated to remove it from an object. Their concept for attitude control is to twist the booms and possibly shift the center of mass.

Both hardware development activities began with a 3-mo concept study for a design reference mission. This was followed by a 12-mo phase to design, fabricate, and test $10-\mathrm{m}$ subscale solar sails to drive out design issues in the spring of 2004. The next phase focuses on scaling the design and fabrication processes followed in early 2005 by the demonstration of a 20-m subscale solar sail system at the Plum Brook facility at GRC near Sandusky, OH. 


\section{Recent Accomplishments}

In the summer of 2003, solar sail technology was selected as one of the candidate technologies for the New Millennium Program ST-9 mission. In January 2004, LaRC presented a conceptual design for an optical diagnostic system (ODS) to provide on-orbit characterization of solar sail shape. An ODS breadboard exercise was conducted in conjunction with the following activity. In March and April 2004, the 10-m system ground demonstrators were tested in NASA's thermal vacuum chambers. Tests included deployment functionality demonstrations and static and dynamic structural characterizations.

\section{Advanced Chemical Propulsion}

\section{A. General Description}

Though it has its limitations, chemical propulsion will be the workhorse of in-space transportation for many years to come. Those technologies that reduce the mass fraction of the propulsion system in favor of science will be the focus of in-space propulsion technology investments in this area.

\section{B. Technical Approach}

Planned investments in this area are currently under review.

\section{Solar Thermal Propulsion}

\section{A. General Description}

Solar thermal propulsion makes use of sunlight to directly heat and expel a propellant, in lieu of either chemical combustion or elecromagetic acceleration of it. The heated propellant is fed through a conventional rocket nozzle to produce thrust. The engine thrust is directly related to the surface area of the solar collector and to the local intensity of the solar radiation.

\section{B. Technical Approach}

Most proposed designs for solar thermal rockets use hydrogen as their propellant due to its low molecular weight, but many other propellants are being considered. There are two basic solar thermal propulsion concepts, differing primarily in the method by which they use solar power to heat the propellant.

Indirect solar heating involves pumping the propellant through passages in a heat exchanger that is heated by solar radiation. The windowless heat exchanger cavity concept is a design taking this radiation absorption approach.

Direct solar heating involves exposing the propellant directly to solar radiation. The rotating bed concept is one of the preferred concepts for direct solar radiation absorption; it offers higher specific impulse than other direct heating designs by using a retained seed (tantalum carbide or hafnium carbide) approach. The propellant flows through the porous walls of a rotating cylinder, picking up heat from the seeds, which are retained on the walls by centrifugal force. The carbides are stable at high temperatures and have excellent heat transfer properties.

\section{Momentum Exchange Electrodynamic Reboost Tethers}

\section{A. General Description}

Momentum-exchange tether propulsion transfers momentum from one object to another by briefly linking a slow-moving object with a faster one. Much the same way ice skaters play "crack the whip," the slower object's speed could be dramatically increased as momentum and energy is transferred to it from the faster object. Similarly, a rotating tether facility in an elliptical Earth orbit might snare slower moving spacecraft in low-Earth orbit and throw them into much higher energy orbits.

The momentum-exchange electrodynamic reboost (MXER) tether propulsion system could use momentumexchange to transfer satellites from low-Earth orbit to geosynchronous transfer orbit and beyond. After throwing the payload, the MXER tether would then use energy collected from solar panels to drive electrical current through the tether. This electrical current would push against the Earth's magnetic field and reboost the tether's orbit, restoring the energy that was transferred to the payload.

Once launched to low-Earth orbit by a rocket, the MXER tether system is intended to deploy a tether roughly 60 to $90 \mathrm{mi}(100$ to $150 \mathrm{~km})$ in length. This tether would be made of lightweight, high-strength material, coated 
for protection from the space environment. Part of the tether would also incorporate an insulated conductive material, like aluminum, to carry the electrical current needed to reboost the tether.

Payloads bound for high-Earth or escape orbits, such as communications satellites and interplanetary spacecraft, would be launched by rocket to low-Earth orbit. There, they would rendezvous with the tip of the spinning MXER tether, which would snare them via a net-like catch mechanism and then throw them toward their final destination. The process would eliminate or reduce the need for the upper-stage booster rocket usually associated with these high-energy orbits. And because the MXER tether could reboost its own orbit without propellant, it could repeat its orbital transfer duties for the duration of its lifespan. Also, since the need for an expendable upper-stage rocketnormally used only once-is eliminated or reduced, this capability would mean smaller, less expensive rockets could be used to launch the payloads.

\section{Technology Planning and Systems Analysis}

\section{A. General Description}

The In-Space Propulsion Technology Program established a technology planning and systems analysis team to identify technology investment needs. The main objective of the systems analysis team is to support the program with appropriate systems analysis studies to provide critical data to use in determining funding priorities and program direction. The In-Space Propulsion Technology Program has assembled a core systems analysis team that can support quick turnaround studies and will create and/or utilize a database of tools and simulations in each technology area to support systems analysis studies.

\section{Conclusion}

Progress is being made toward the delivery of high-priority, first- and second-generation in-space propulsion technologies for potential space science applications within the next 2-3 yr. The next-generation electric (ion) propulsion system is moving toward an integrated ground demonstration in FY 2006. The first generation rigid aeroshell is comparably headed toward ground validation in the same timeframe. Solar sail technology, while impossible to completely validate to TRL 6 on the ground, will be matured sufficiently for flight validation as early as FY 2006/2007. 